\title{
Sampling Frequency and Time Window Influence on Flicker Measurements Significance. A Case Study.
}

\author{
F. Oliveira ${ }^{1,2}$, A. Madureira ${ }^{3}$ and M. P. Donsión ${ }^{4}$ \\ ${ }^{1}$ School of Technology and Management, Polytechnic Institute of Leiria \\ Morro do Leno - Alto Vieiro, Apartado 4163, 2411-901 Leiria, Portugal \\ Phone: +351 244 820300, Fax:+351 244 820310, E-mail: ftadeu@estg.ipleiria.pt \\ 2 INESC Coimbra \\ Rua Antero de Quental, Nº199, 3000 - 033 Coimbra, Portugal \\ ${ }^{3}$ Power Systems Unit of INESC Porto \\ Rua Dr. Roberto Frias, 378, 4200-465 Porto, Portugal \\ Phone: +351 22 2094000, Fax:+ 35122 2094050, E-mail: agm@inescporto.pt \\ ${ }^{4}$ Department of Electrical Engineering, E.T.S.I.I., University of Vigo \\ Campus of Lagoas - Marcosende, 36310 Vigo, Spain \\ Phone: +34 986 812685, Fax:+34 986 812601, E-mail: donsion@uvigo.es
}

\begin{abstract}
Most of the Power Quality surveys in industrialized countries are performed according to the parameters defined in the EN50160 standard, which sets oneweek measurement periods with ten-minute sampling time for most power quality disturbances such as flicker and harmonics. Both the sampling periods and the measurement period have a great influence on the significance of the values obtained as well as on the conclusions taken. In this paper, an attempt is made to compare the values obtained with different sample frequencies and time windows, working on instantaneous flicker (Ifl) values for a wind turbine over three weeks.
\end{abstract}

\section{Key words}

Power Quality, Flicker, Wind Farms, Sampling Frequency, Time Window.

\section{Introduction}

The main goal of this paper is to establish a comparison between power quality disturbance parameters measured using different sampling frequencies and time windows.

The present study is based on a power quality measurement campaign which took place in the Sotavento Experimental Wind Farm (SEWF) in Galicia, Spain, with special focus on two particular types of disturbances: Flicker and Harmonics. Harmonics analysis is, however, out of the scope of this paper.

\section{Field Measurements in the SEWF}

Inaugurated in June 2001, the SEWF presents an innovative concept, contemplating two different lines of action: one, technical-commercial and other, educationalformative.

The SEWF is located in Galicia, northwest of the Iberian Peninsula, at an altitude of $600 \mathrm{~m}$ between the Spanish provinces of La Coruña and Lugo. The average wind speed is $7.5 \mathrm{~m} / \mathrm{s}$, with predominant wind directions $\mathrm{SW}$ and NE.

Its main distinctive feature is the large degree of technological diversity, with five different generation models. It holds a total of 24 wind turbines, 3 of which are prototypes. It has a total of $17.56 \mathrm{MW}$ installed capacity, with an annual estimate production of 38500 MWh.

The measurement campaign took place over three weeks, at three measuring sites, in two different wind turbines and in the wind farm substation. The measuring equipments used were one 'MEMOBOX 300 smart' unit and two 'Unilyzer 812' units.

The 'MEMOBOX 300' and the 'Unilyzer 812' perform power quality measures according to international standards applicable to the system quantities, and analyse results according to European standard EN 50160. Both are capable of gathering and appropriately storing voltage harmonics and flicker, among other power quality indices.

For this particular study, the data eventually selected for analysis and presentation was obtained in wind turbine $n$. 4, an ECOTECNIA 44/640, on phase 1. 


\section{Flicker Measurement}

According to EN 50160, Voltage Fluctuation is defined as a series of voltage changes or a continuous variation of the root-mean-square voltage value. These fluctuations may be caused by load variation or by generation change based on intermittent power sources, such as wind turbines. Therefore, wind generation contributes to voltage fluctuations that are perceived by consumers in the network and impacts strongly on power quality.

Generally, voltage fluctuations that do not exceed $\pm 10 \%$ of the nominal voltage value do not affect significantly most electrical equipments. However, some voltage fluctuations can cause a nuisance, visually perceptible effect called flicker.

Also according to EN 50160, Flicker is defined as an impression of unsteadiness of visual sensation induced by a light stimulus, the luminance or spectral distribution of which fluctuates with time. Despite this definition, flicker can also affect electric appliances than lighting equipment and have consequences other than visual distress.

Flicker severity is the intensity of flicker annoyance as defined by the IEC flicker measuring method (defined in IEC standard 61000-4-15) and evaluated by the following quantities:

- Short term severity $\left(\mathrm{P}_{\mathrm{st}}\right)$ measured over a period of ten minutes.

- Long term severity $\left(\mathrm{P}_{1 t}\right)$ calculated from a sequence of $12 \mathrm{P}_{\mathrm{st}}$ - values over a two-hour interval, according to the following expression:

$$
P_{l t}=\sqrt[3]{\sum_{i=1}^{12} \frac{P_{s t_{i}}{ }^{3}}{12}}
$$

Flicker depends mostly on frequency, amplitude and duration of the voltage fluctuations that originate it. The frequencies associated to these effects are in the range of $0,5 \mathrm{~Hz}$ to $30 \mathrm{~Hz}$, and visual perception is usually highest for $8 \mathrm{~Hz}$ to $10 \mathrm{~Hz}$ fluctuations. IEC standard 61000-4-15 presents a blocks diagram of equipment adequate to the measurement of flicker.

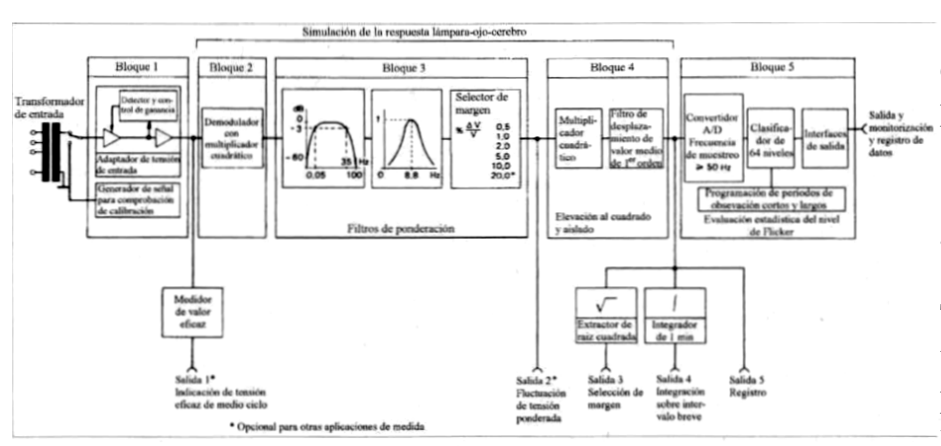

Fig. 1. Block diagram of the flickermeter defined in IEC 61000-4-15

\section{Sample Frequency and Time Window Influence - the EN50160 Parameter Definition}

Nowadays nearly all power quality measurement campaigns are performed in accordance with the parameters stated on the EN50160 European standard. While this standardization of processes can have very positive effects where comparability of results and installations is concerned, the truth is that such tendency raises three important questions.

The first is that the EN50160 standard specifies limits as well as measuring protocols but it only applies to normal operating conditions, since the document specifies a number of situations to which the limits defined are not applicable. A description of the Electromagnetic Environment should include all events that may occur to a consumer and not only variations that may happen under normal operating conditions.

A second question, closely related to the first, concerns the fact that all across Europe, generating companies are characterizing their power quality by measuring according to the EN50160: 10-minute voltage averages, 10 -minute harmonic voltages measurements, etc. The values that do not exceed the limits within $95 \%$ of the measuring time are used to characterize power quality at the site. The problem arises when some generating companies compare these values to the limits specified in the standard and say that their power quality is compliant with the EN50160 standard. Understanding the concept of voltage characteristics, it is hardly surprising that voltage quality at the site is better than the limits established, since the limits themselves were set using 'worst case scenarios'. Hence, this standard should never be used to certify power quality.

The third question, and the one which will be specifically addressed in this paper, concerns both the measurement period and the sampling frequencies for the disturbances measured. The ten-minute standard period is probably adequate for most consumer installations with a 'smooth' behaviour (even those with severe power quality disturbance problems), but not if the electric behaviour is irregular, as in the case of wind power generation. Also, in other cases, like the case studied here, such a comparatively long time between two consecutive samples may conceal occasional yet significant levels of disturbance, which can have a strong impact on overall power quality.

\section{Studies, Results and Discussion}

The raw data used for this case study was obtained in a three consecutive week measurement campaign. Several power quality parameters were measured on a number of wind turbines, but for this paper the highlight is on short and long term flicker values (Pst and Plt) and instantaneous flicker (Ifl) values, logged every second. 
Due to the large amount of data available, a specific wind turbine and a phase were selected, both for the sake of simplicity and comparability.

\section{A. Sampling Frequency/Period Influence}

In order to observe the effect of different sampling frequencies, the standard one-week measurement period was observed, and the data was sorted and filtered, thus obtaining:

- Ifl values, 1 second sampling period (Ifl1sec);

- Ifl values, 1 minute sampling period (Ifl1 min);

- Ifl values, 10 minutes sampling period (Ifl10min);

- $\quad$ Pst, 10 minutes calculation period.

At this point, it is important to observe that Pst, while being calculated every 10 minutes, results of several measurements taken in considerably shorter periods of time. In fact, the difference between the 10-minute sampled values of Ifl and the values for Pst (also taken every 10 minutes) is significantly different. This has mostly to do with the algorithm followed to calculate Pst, which takes into account a number of percentiles.

A first approach to comparing the different sampling periods is a time plot, and some were created. Notably, this approach is partially limited by the number of points to plot, which can go up to 600000+ for 1-week measurement with 1 -second sampling period.

As an example, we shall compare the time plot of Ifl1sec with the Pst chart, for a 2-hour period:

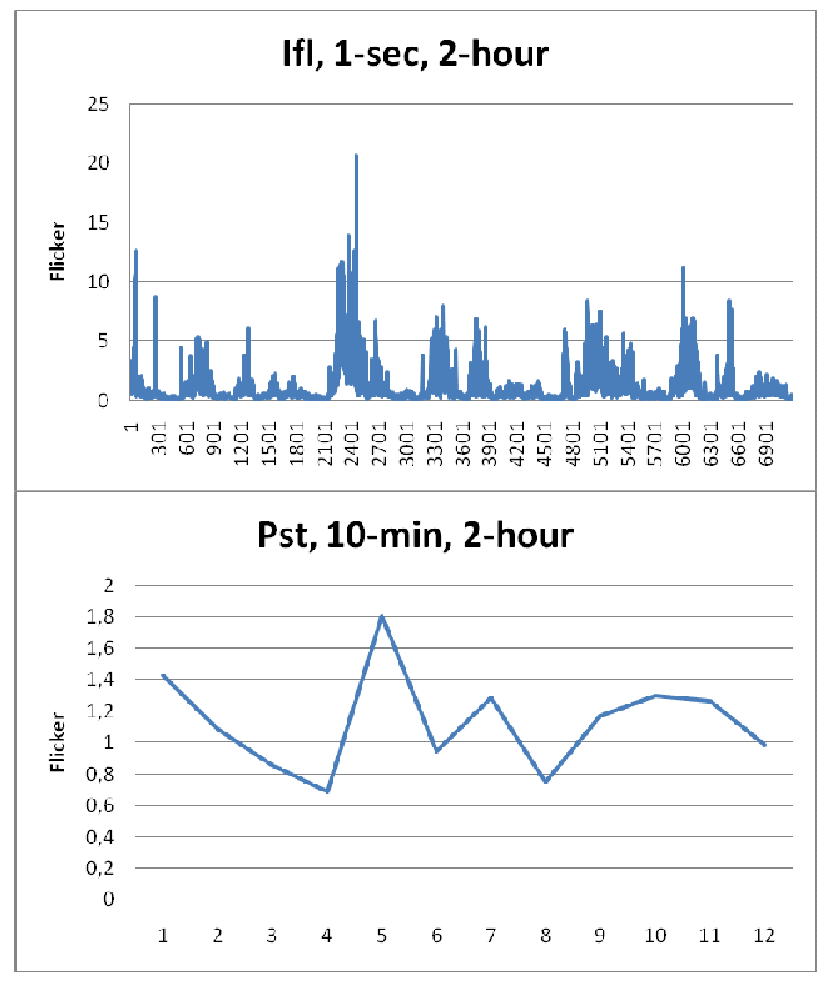

Figs. 2 and 3. Time plots of Ifl $1 \mathrm{sec}$ and Pst for a 2-hour period.
It is important to state at this point that both Ifl and Pst measured values are obtained via the IEC 61000-4-15 flickermeter model, the Pst values being obtained by applying a specific algorithm.

Comparing the two plots in figure 2 , it is patently clear that the differences are deeper than the mere number of points. In fact, the Pst index clearly 'smooths' and 'scales' the instantaneous flicker values. While this calculation method for the Pst is legitimated by both visual distress studies and international standards, it is also true that it is based merely on the interpretation of the visual effect of voltage fluctuations, and not explicitly on possible quality disturbances on electric devices.

In order to test a wide range of situations, many time periods were tested. Results obtained are presented both graphically and in table form, for three periods of time, where each larger period includes every smaller one. In this particular case, percentiles 95 and 99 are presented rather than maximum values, sometimes considerably superior, in an effort to filter any isolated incident or possible measurement error.

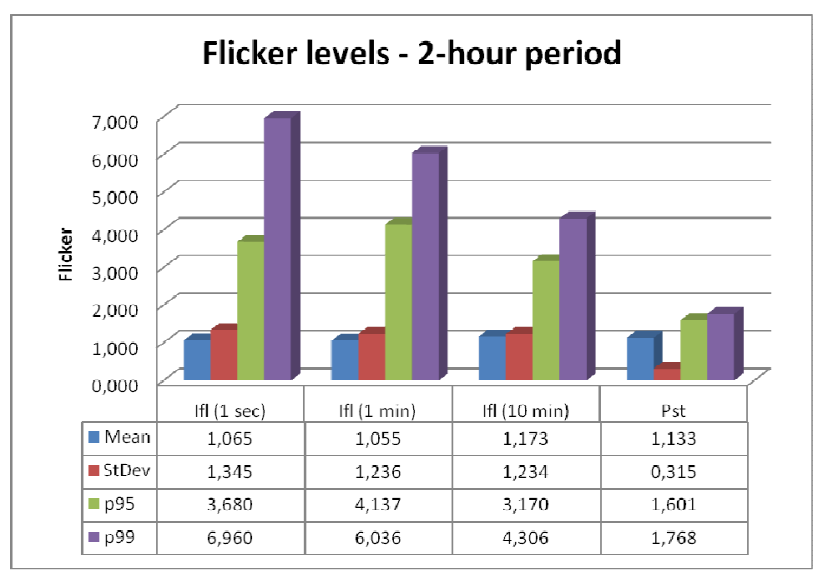

Fig. 4. Flicker levels over a 2-hour period: Mean, Standard Deviation and Percentiles 95 and 99 for various sampling periods

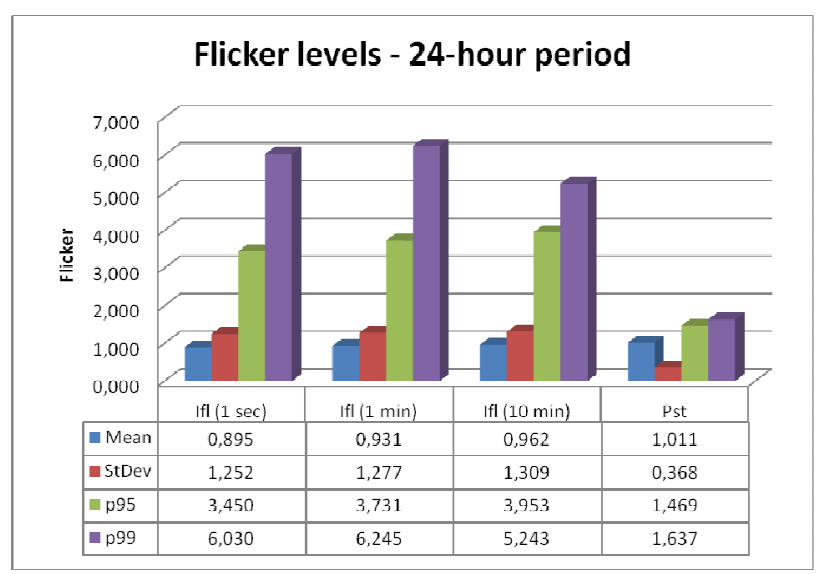

Fig. 5. Flicker levels over a 24-hour period: Mean, Standard Deviation and Percentiles 95 and 99 for various sampling periods 


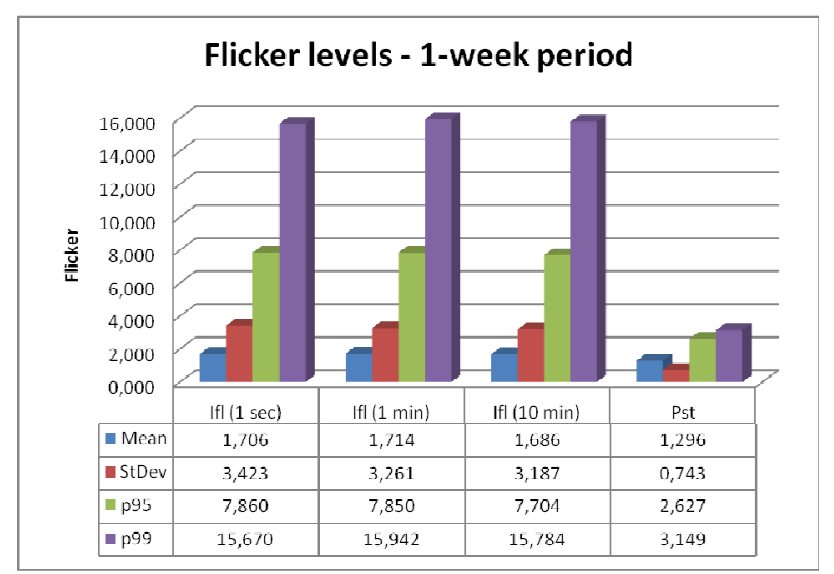

Fig. 6. Flicker levels over a 1-week period: Mean, Standard Deviation and Percentiles 95 and 99 for various sampling periods

On comparison, and based on the information provided in Figs. 4, 5 and 6, it is visible that the Pst level is quite similar to the mean value. The calculation algorithm used justifies the lower standard deviation, and thus the comparative 'stability' of the index along different periods of time. Again, this goes to show that Pst is a good estimator of the nuisance level caused by flicker only if short-term instantaneous flicker values can be disregarded, or at least given lower importance.

Also, now comparing only the Ifl values, another expected result is found in the fact that a higher sampling frequency is progressively less important when a longer measurement period is considered.

\section{B. Time Window Influence}

In the previous sub-section, some considerations were already derived concerning the time window influence, since three different time periods are considered. However, an accurate study of time window influence should be based on data on periods longer than the one week specified in EN50160, for the reasons presented in section 4 .

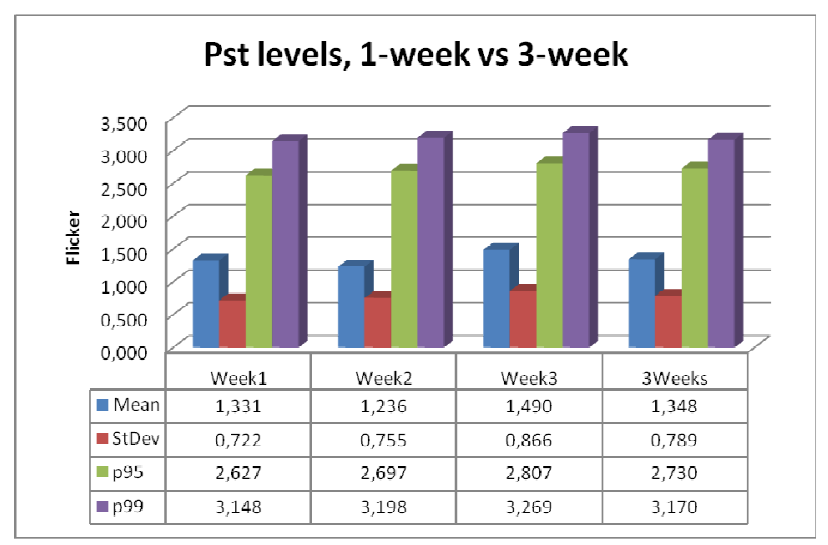

Fig. 7. Flicker levels over 1-week and 3-weeks periods: Mean, Standard Deviation and Percentiles 95 and 99
In this study, and due to the time span limitations of the data, only minor results can be obtained and discussed. Fig. 7 shows that little or no advantage is gained from tripling the time window. Yet, and although absent in the chart, absolute maximum levels vary, reaching 12,75 in the last week, as compared to around 3,65 in the previous two weeks. Studies were also performed using Ifl data, providing similar results.

Still, it is quite clear that, in order to obtain consequences on the time window influence on flicker measurements, longer periods must be considered, or at least weeks in different year seasons.

\section{Conclusions and Future Work}

The results obtained and discussed in the previous section confirm the premise that typical parameters used when performing surveys may be provide incomplete or imprecise information, whenever electric installations with irregular behaviour are considered. Following such premise, other parameters and methodologies may be developed to enhance the perception on the surveyed facilities.

In particular, generating installations like wind farms must be assessed in a different way and this is partially covered by IEC $61400-21$. Other standards, or at least new guidelines may be necessary to adequately deal with power quality measurement campaigns in these types of installations.

Since these conclusions are based on a single case study, future work on the subject should include more measurement campaigns in different places, with longer measurement periods (up to one year). On the other hand, other power quality disturbances, such as harmonics, may be analysed in order to determine if currently used parameters are adequate.

\section{Acknowledgement}

The financial support from the "Ministerio de Educación y Ciencia" and FEDER funds, ENE2007-68032-C0401/CON Project, is gratefully acknowledged.

\section{References}

[1] EN 50160, "Voltage characteristics of electricity supplied by public distribution systems", 1999.

[2] IEC Standard 61000-4-15, "Electromagnetic compatibility (EMC) - Part 4: Testing and measurement techniques - Section 15: Flickermeter Functional and design specification", 2003.

[3] F. Oliveira, A. Madureira, M.P. Donsión, "Experimental Study of Power Quality in Wind Farms”, ICREPQ' 04, 2004.

[4] A. Madureira, F. Oliveira, M.P. Donsión, "Statistical Study of Power Quality in Wind Farms", ICREPQ' 04, 2004. 
[5] F. Oliveira, A. Madureira, M.P. Donsión, "Power Quality Impact of Wind Farms on Domestic and Industrial Facilities", ICREPQ’05, 2005.

[6] M. Bollen, "Understanding Power Quality Problems: Voltage Sags and Interruptions", IEEE, New Jersey: John Wiley \& Sons, Inc., 2000. 